\title{
Measurement of Open Heavy Flavor Production in the STAR Experiment at RHIC
}

\author{
Wei Xie for the STAR Collaboration ${ }^{1}$ \\ Dept. Of Physics, Purdue University \\ 525 Northwestern Ave., West Lafayette, IN 47907, US \\ E-mail: wxielpurdue.edu
}

\begin{abstract}
Heavy quarks are unique probes to study the properties of the hot and dense medium created at RHIC. Unlike light quarks, heavy quark masses come mostly from spontaneous symmetry breaking, which makes them ideal for studying the medium's QCD properties. Due to their large masses, they are produced in the early stages of the collisions and are expected to interact with the medium quite differently from that of light quarks. Measurements of the open heavy flavor production in the $p+p$ collisions not only are crucial baseline references for understanding the medium effect of heavy quark productions in heavy-ion collisions, but also provide crucial tests on the QCD framework. In this paper, we present the results of open heavy flavor production in the STAR experiment at RHIC, focusing on the non-photonic electron measurement in $p+p$ collisions at $\sqrt{s}=200 \mathrm{GeV}$. We also describe briefly the future perspective of open heavy flavor measurements in the STAR experiment.
\end{abstract}

XVIII International Workshop on Deep-Inelastic Scattering and Related Subjects - DIS 2010

Firenze, Italy

April 19-23 201

1 Wei Xie 


\section{Introduction}

Detailed studies of the heavy quark production in the strongly coupled QGP created at RHIC provide crucial information in understanding its properties. The STAR experiment at RHIC is a large acceptance, multipurpose experiment, where open heavy flavor can be measured directly through their hadronic decay channels [1], or indirectly through their semileptonic decay [2]. Our direct measurements are restricted in low momentum region due to the lack of precise secondary vertex tracking, while the alternative indirect measurements allow us to study heavy quark production in low $\mathrm{p}_{\mathrm{T}}$ as well as the high $\mathrm{p}_{\mathrm{T}}$ region with the deployment of fast online triggers.

Despite the fact that the heavy quark physics is still at its early stage of investigation at RHIC, current measurements have already posed serious challenges to the understanding of heavy quark production in the medium. Large suppression of high $\mathrm{p}_{\mathrm{T}}$ heavy flavor decay electron (non-photonic electron) production was discovered through measuring the nuclear modification factor $\left(\mathrm{R}_{\mathrm{AA}}\right)$ in $200 \mathrm{GeV}$ central $\mathrm{Au}+\mathrm{Au}$ collisions [2], [3]. This has triggered a lot of activities in the field trying to understand its origin [4]. Although the $\mathrm{R}_{\mathrm{AA}}$ measurements from STAR and PHENIX are consistent with each other, the measured high $\mathrm{p}_{\mathrm{T}}$ non-photonic electron invariant yield in both $p+p$ and $\mathrm{Au}+\mathrm{Au}$ collisions differ by about a factor of two. Furthermore, large differences are observed in the measured heavy quark total cross section between PHENIX and STAR which, in additional to non-photonic electrons, includes measurements of low $\mathrm{p}_{\mathrm{T}} \mathrm{D}^{0}$ and single muons [5], indicating that the discrepancy also appears in low $\mathrm{p}_{\mathrm{T}}$ region. Solving these discrepancies is important since they not only influence our understanding of the medium responses to heavy quarks but also complicate the interpretation of heavy quarkonium measurements at RHIC.

In this paper, we first report the progress in solving the discrepancy of high $\mathrm{p}_{\mathrm{T}}$ nonphotonic electron measurements in $200 \mathrm{GeV} p+p$ collisions. Then we describe how to disentangle electrons from bottom and charm hadron decays. At the end, we provide summary and outlook.

\section{Results and Discussion}

\subsection{Invariant Cross Section of Non-photonic Electron Production in $p+p$ Collisions}

The dominant background of early STAR measurement using the run 2003 data is the large amount of conversion electrons originating from the silicon detectors. To significantly reduce the background of this measurement, starting from the 2008 run, STAR removed the silicon detectors. We measure the non-photonic electron invariant cross section at $\sqrt{s}=$ $200 \mathrm{GeV}$ using the data from the low material $2008 p+p$ run as well as the 2005 run with silicon detector installed as a cross check. In both runs, STAR sampled an integrated luminosity of about $2.7 \mathrm{pb}^{-1}$, which is much higher than that of 2003 run. Note that in these analyses, we do not subtract electrons from heavy quarkonia and Drell-Yan decays.

The left panel of Figure 1 shows the ratio of non-photonic electron over photonic electron yield as a function of $\mathrm{p}_{\mathrm{T}}$ obtained from the 2008 and 2005 data analyses. The ratio in the 2008 
run is much higher due to much less material thickness in front of TPC after removing the silicon detectors. The right panel of Figure 1 shows the non-photonic electron invariant cross section $\left(\left(e^{+}+e^{-}\right) / 2\right)$ as a function of $\mathrm{p}_{\mathrm{T}}$ in $p+p$ collisions from the analyses of the 2008 and 2005 runs. Despite the large difference in photonic background, the two new measurements agree with each other very well.
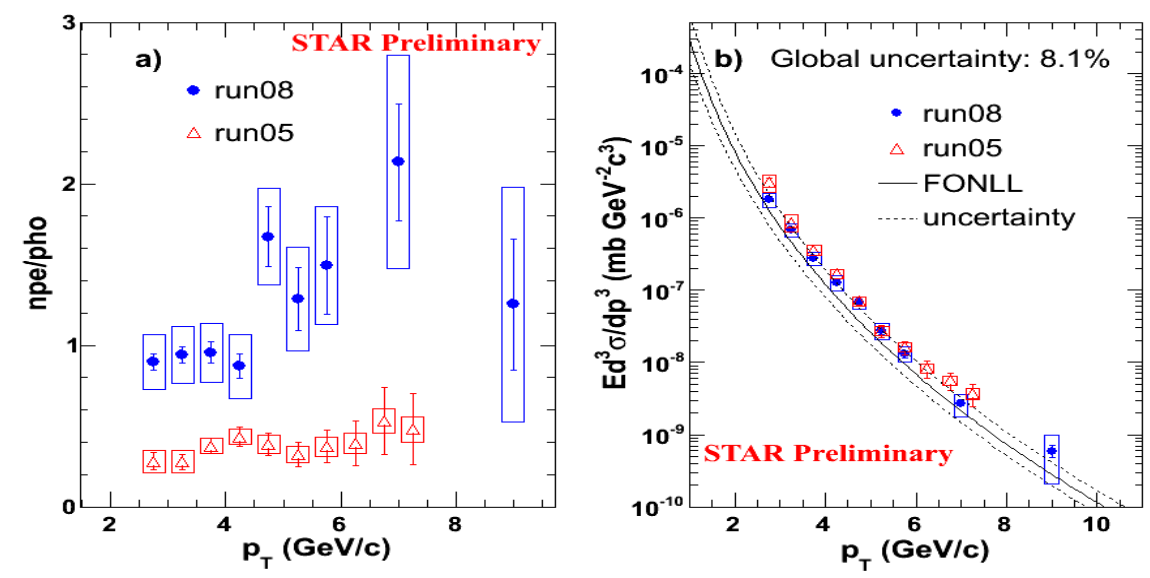

Figure 1: (a) Ratio of non-photonic electron over photonic electron as a function of $\mathrm{p}_{\mathrm{T}}$ from the 2008 (closed circles) and 2005 (open triangles) analyses. (b) Invariant cross section of non-photonic electron $\left(\frac{e^{+}+e^{-}}{2}\right)$ as a function of $\mathrm{p}_{\mathrm{T}}$ from the 2008 (closed circles) and 2005 (open triangles) analyses .

To further clarify the consistency between the two results, we compare the ratio of each individual measurement over FONLL calculation as shown in Figure 2, where the red closed circles are the result of combining the 2008 and the 2005 measurements at $\mathrm{p}_{\mathrm{T}}<6 \mathrm{GeV} / \mathrm{c}$ through "Best Linear Unbiased Estimate" (BLUE) method [6]. The $\chi^{2}$ test from the "BLUE" methods shows that the $\chi^{2} / \mathrm{NDF}$ of our 2008 and 2005 measurements is 0.65 . This means the two results are consistent with each other.

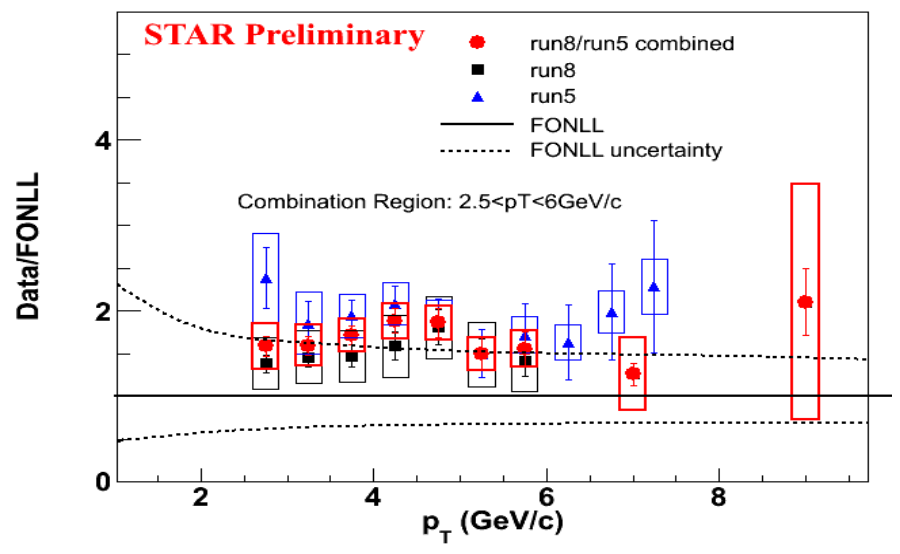

Figure 2: Ratio of the 2008 (closed squares), the 2005 (closed triangles) measurements and the results combining the two analysis (closed circles) over FONLL calculations.

Figure 3(a) shows the comparison of the STAR measured invariant cross section combining the 2008 and 2005 data, with the early published STAR result obtained from 2003 
run and PHENIX result. Figure 3 (b) shows the ratio of each individual measurement in (a) over the FONLL calculations. One can clearly see that STAR new results and PHENIX published result are consistent with each other within the quoted uncertainties at $\mathrm{p}_{\mathrm{T}}>2.5 \mathrm{GeV} / \mathrm{c}$. The problems in the analysis that leads to our early publication are under investigation. The upper limit of FONLL calculation is consistent with this measurement.
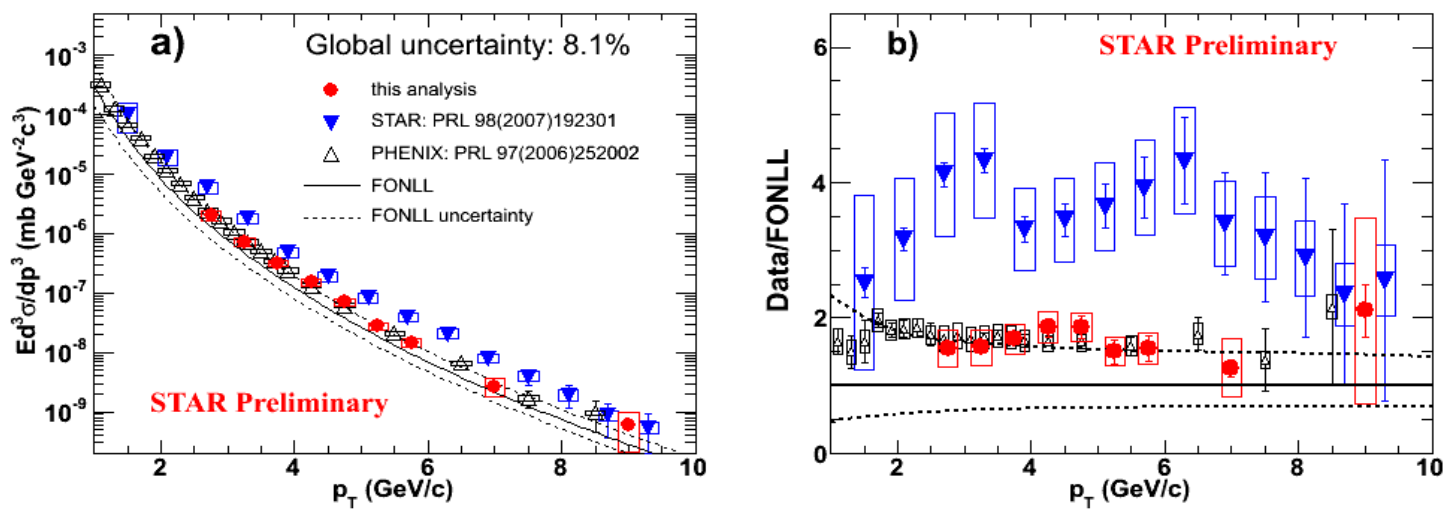

Figure 3: (a) Invariant cross section of non-photonic electron from this analysis combining the 2008 and 2005 data (closed circles), STAR published result using year 2003 data (closed triangles) and PHENIX published results (open triangles). FONLL and its uncertainties are shown in solid and dashed lines. (b) Ratio of each individual measurement shown in (a) over FONLL calculations.

\subsection{Disentangle Electrons from Charm and Bottom Meson Decays}

The relative contribution to the non-photonic electrons from bottom and charm meson decays can be disentangled utilizing their different decay kinematics. In STAR, this is done though measuring the azimuthal correlation between non-photonic electrons and charged hadrons (e-h) as well as the correlation between non-photonic electrons and $D^{0}\left(\mathrm{e}-\mathrm{D}^{0}\right)$ [7].
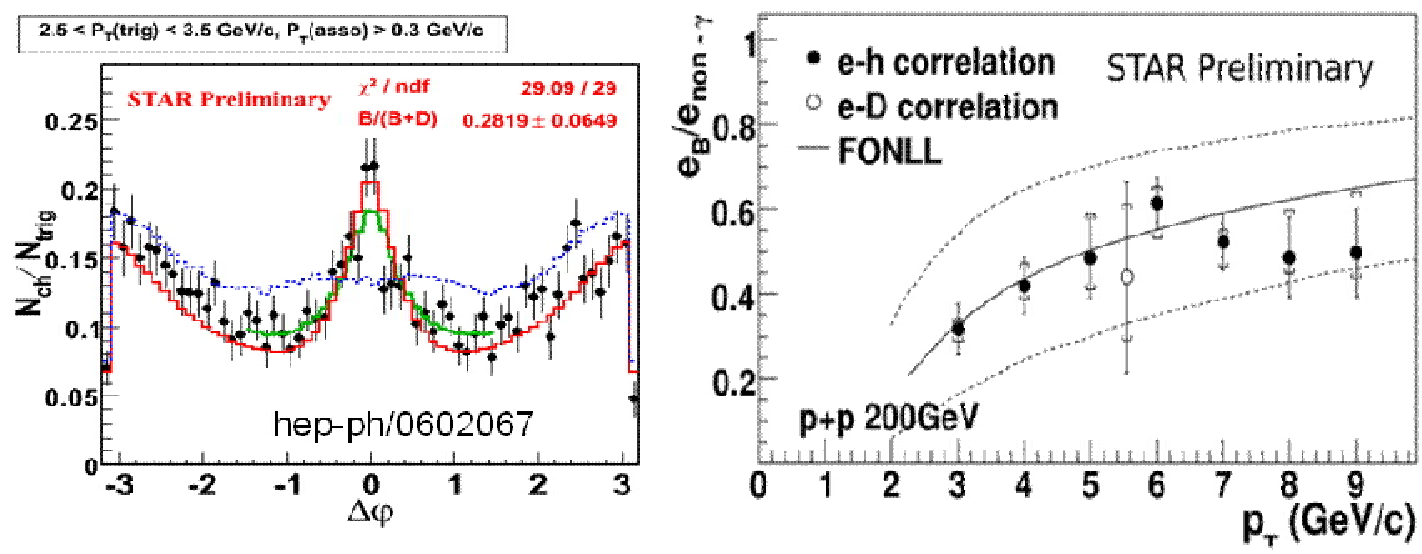

Figure 4: (Left panel) Distribution of the azimuthal angle between non-photonic electrons and charged hadrons with the associated hadron yields normalized to per trigger electron (closed circles) at $2.5<\mathrm{p}_{\mathrm{T}}$ (electron) $<3.5 \mathrm{GeV} / \mathrm{c}$, together with the simulated result for electrons decay from bottom (blue) and charm meson (red) as well as a fit combining the two components (green curve). (Right panel) Relative contribution of bottom meson to non-photonic electron yield as a function of the $\mathrm{p}_{\mathrm{T}}$ using e-h (closed circles) and e- $\mathrm{D}^{0}$ (closed squares) correlations. The error bars and brackets represent statistical and systematic uncertainties, respectively. 


\section{Summary and Outlook}

The measurements of non-photonic electron invariant cross section from STAR and PHENIX experiment are consistent with each other within the quoted uncertainties at $\mathrm{p}_{\mathrm{T}}>$ $2.5 \mathrm{GeV} / \mathrm{c}$. The problems in our early published result are under investigation. The upper limit of FONLL calculation is consistent with this measurement. We found that more than $30 \%$ nonphotonic electrons at $\mathrm{p}_{\mathrm{T}}>3 \mathrm{GeV} / \mathrm{c}$ come from bottom meson decay in $200 \mathrm{GeV} p+p$ collisions. In the 2009 and 2010 run, beside the installation of the full TOF detector, STAR accumulated high quality data samples with large integrated luminosity in both $p+p$ and $\mathrm{Au}+\mathrm{Au}$ collisions. We expect statistically significant measurement of direct D meson and the non-photonic electron in $p+p$ and $A u+A u$ collisions, which should provide good constraint on the total heavy quark production cross section and their energy loss in the hot and dense medium. In the near future, with the RHIC-II luminosity upgrade, the installation of STAR Heavy Flavor Tracker and Muon Telescope Detector, we expect high precision heavy quark measurements that will provide us crucial information to further understand the strongly coupled QGP at RHIC.

\section{References}

[1] B. I. Abelev et al. [STAR Collaboration], Phys. Rev. D 79, 112006 (2009); S. Baumgart, Eur. Phys. J. C 62, 3 (2009); J. Adams et al. [STAR Collaboration], Phys. Rev. Lett. 94, 062301 (2005).

[2] B. I. Abelev et al. [STAR Collaboration], Phys. Rev. Lett. 98, 192301 (2007).

[3] A. Adare et al. [PHENIX Collaboration], Phys. Rev. Lett. 97, 252002 (2006); S. S. Adler et al. [PHENIX Collaboration], Phys. Rev.Lett. 96, 032301 (2006).

[4] M. G. Mustafa, D. Pal, D. K. Srivastava and M. Thoma, Phys. Lett. B 428, 234 (1998); M. Djordjevic, M. Gyulassy, R. Vogt and S. Wicks, Phys. Lett. B 632, 81 (2006); S. Wicks, W. Horowitz, M. Djordjevic and M. Gyulassy, Nucl. Phys. A 784, 426 (2007); M. Djordjevic and U. W. Heinz, Phys. Rev. Lett. 101, 022302 (2008); R. Sharma, I. Vitev and B. W. Zhang, Phys. Rev. C 80, 054902 (2009).

[5] B. I. Abelev, et al, [STAR Collaboration], arXiv:0805.0364 [nucl-ex].

[6] A. Valassi, Nucl. Instr. Meth. A 500, 391 (2003).

[7] B. Biritz for STAR Collaboration, Nucl. Phys. A 830, 849c (2009) 